\title{
Transition Enabling Petri Nets to Supervisory Control Theory
}

\author{
G.C. Barroso ${ }^{\dagger}$, A.M.N. Lima $a^{\ddagger}$ and A. Perkusich \\ ${ }^{\dagger} D F / U F C,{ }^{\ddagger} D E E / U F P B$ \\ Av. Aprígio Veloso, 882 - 58109-970 - Campina Grande - PB - Brazil \\ FAX +55833332480 - e-mail: $\{$ gcb, marcus, perkusic\}@dee.ufpb.br
}

\begin{abstract}
This paper presents a Petri Net (PN) model conceived to be employed with the Supervisory Control Theory (SCT). The supervisor synthesis is obtained by processing both the system and specification models through the proposed algorithms. These algorithms make possible to obtain the controller of a discrete event system based on a given specification. Its simplicity and efficiency are demonstrated using a typical manufacturing cell problem.
\end{abstract}

\section{Keywords}

Supervisory control, discrete event systems, Petri nets, manufacturing systems.

\section{INTRODUCTION}

Over the last years the use of the Supervisory Control Theory (SCT) in the design of controllers for Discrete Event Systems (DES), has rapidly increased. Among other formalisms, Petri nets (PN) (Murata, 1989) have been widely used as a powerful tool in the analysis and design of discrete event systems (Giua and DiCesare, 1994; Sreenivas, 1993). In general, the controller synthesis is achieved by changing the model structure. In most cases the structure modification does not follow any standard procedure.

This paper investigates a new approach to the supervisors synthesis, using the SCT and an extension of a class of Petri nets, called Petri Nets with Transition Enabling Functions $(P N T E F)$ (Papelis and Casavant, 1992). Two new algorithms are introduced in order to synthesize the controller of a DES.

The present paper is organized as follows: In Section 2 the the basic definition of the SCT is presented. In Section $3 P N T E F$ s are defined. In Section 4 the proposed algorithms to synthesize the discrete event controller are outlined. In Sections 5 and 6 two examples are discussed to exemplify the proposed approach. And finally in Section 7 the main conclusion of the paper are presented. 


\section{GENERAL DEFINITIONS}

\subsection{Supervisory Control Theory}

In the SCT (Ramadge and Wonham, 1989), the system behavior is represented by a 5 tuple $G=\left(\sum, Q, \delta, q_{0}, Q_{m}\right)$, called generator. The symbol $\sum$ represent the set of event labels, or event alphabet; $Q$ is a set of states; $\delta: \sum \times Q \rightarrow Q$ is the transition function defined at each $q \in Q$, so that $\delta(\sigma, q)$ is defined only to some subset $\sum(q) \subset \sum$ that depends on $q$. The marked states $Q_{m}$ represent the end of an event sequence, and also represent the execution of a task by the system. The set of all strings formed by any number of symbols from $\sum$, including the empty symbol $\epsilon$, is denoted by $\Sigma^{*}$. The transition function $\delta$ is extended to process strings from $\sum^{*}$.

A state $q$ is said to be accessible iff $\exists s \in \Sigma^{*} \mid \delta\left(s, q_{0}\right)=q$. A state $q$ is said to be coaccesible iff $\exists s \in \Sigma^{*} \mid \delta(s, q) \in Q_{m}$. G is trim iff it is accessible and coaccessible.

Each generator $G$ has two associated languages: $L(G)$ is the language generated by $G$ and $L_{m}(G)$ is the language marked by $G$. These languages are sets of words defined on $\sum$. The language $L(G)$ represents the physically possible behavior of the system, while $L_{m}(G)$ represents the tasks it is able to complete.

To control a DES it is necessary to admit that some events may be disabled when desired. To model such control action, the $\sum$ set is partitioned into: i) $\sum_{c}$ the controllable event set and ii) $\sum_{u}$ the uncontrollable event set, such that $\sum=\sum_{c} \cup \sum_{u}$ and $\sum_{c} \cap \sum_{u}=\emptyset$. All the events in $\sum_{c}$ may be disabled at any time, while those in $\sum_{u}$ suffer no influence of an external control action.

A control input for $G$ consists of a subset $\Gamma \subseteq \sum$, satisfying $\sum_{u} \subseteq \Gamma$. If $\sigma \in \Gamma$, then $\sigma$ is enabled by $\Gamma$, otherwise, $\sigma$ is disabled. The condition $\sum_{u} \subseteq \Gamma$ means that the events in $\sum_{u}$ are always enabled.

Let $\Gamma \subseteq 2^{\Sigma}$ denote the set of control inputs. A DES represented by $G$, with a set of control inputs $\Gamma$ is called a controlled DES (CDES). For convenience, one refers to a CDES by its underlying generator $G$.

Controlling a CDES $G$, consists of generating a sequence of elements $\gamma, \gamma^{\prime}, \gamma^{\prime \prime}, \ldots$ in $\Gamma$, in response to the previously observed events generated by $G$. Such a controller will be called a supervisor.

A supervisor is a map $f: L \rightarrow \Gamma$, specifying for each possible string of generated events $w$, the control input $f(w)$ to be applied.

A supervisor is represented by an automaton and an output map, i.e.: $S=\left(\sum, X, \xi, x_{0}\right)$ is an automaton and $\psi: X \rightarrow \Gamma$ is the output map.

One says that the pair $(S, \Psi)$ realizes the supervisor $f$ if for each $w \in L(G / f)$, $\Psi\left(\xi\left(w, x_{0}\right)\right)=f(w)$, where $L(G / f)$ represents the closed behavior of the composed system $G$ supervised by $f$.

The basic supervisory control problem may be stated as follows: Given a DES $G$ with open-loop behavior given by $L$, what closed-loop behavior $K \subseteq L$ can be achieved by supervision? To solve this problem, it is necessary to define the concept of controllability.

Given two arbitrary languages $L, K \subseteq \sum^{*}$ and an alphabet $\sum=\sum_{c} \cup \sum_{\mu}$, one says that $K$ is $L$-closed iff $K=\bar{K} \cap L$, and $\bar{K}$ is L-controllable iff: $\bar{K} \sum_{u} \cap L \subseteq \bar{K}$, where $\bar{K}$ is a prefix of $K$, i.e., the set of all prefixes of the strings in $K$.

Given a generator $G$, the language $K \subseteq L_{m}(G)$ models the desired specification. The language $K$ represents the tasks to be executed under supervision. Then, the goal is to find a proper supervisor $S$ to $G$ so that the closed-loop system satisfies the condition $L(S / G)=K$.

There exists a proper supervisor so that $L(S / G)=K$ iff $K$ is $L_{m}(G)$-closed and $L(G)$ controllable (Ramadge and Wonham, 1989). When these conditions are not satisfied, it is 
always possible to meet a supremal controllable sublanguage $K^{\dagger}$ such that $K^{\uparrow} \subseteq K$. In the case of finite state generators, $K^{\uparrow}$ is always computable (Ramadge and Wonham, 1989).

\section{PNTEF}

A Petri net with transition enabling functions is a 4-tuple $P N T E F=\left(N, l, M_{0}, \Phi\right)$, where: $N=(P, T, I, O)$ is a structure of a place/transition Petri net, where: $P=\left\{p_{1}\right.$, $\left.\ldots, p_{n}\right\}$ is a finite set of places, represented by circles; $T=\left\{t_{1}, \ldots, t_{m}\right\}$ is a finite set of transitions represented by bars or boxes; $P \cap T=\oslash$ and $P \cup T \neq \emptyset . I: P \times T \rightarrow \aleph$ is the input function that specifies the arcs directed from places to transitions $(\aleph=\{0,1,2, \ldots\})$; $O: T \times P \rightarrow \aleph$ is the output function that specifies the arcs directed from transitions to places; $l: T \rightarrow \sum$ is a function that labels the transitions with symbols of the alphabet $\sum ; M_{0}$ is the initial marking; $\Phi=\left(\varphi_{1}, \ldots, \varphi_{m}\right)$ is a set of logical expressions associated with the transitions.

A transition $t_{j}$ is enabled in a $P N T E F$, given a marking $M^{\prime}$, if: $M^{\prime}\left(p_{i}\right) \geq I\left(p_{i}, t_{j}\right), \forall p_{i} \in$ $P$; the logical expression $\varphi_{j}$ associated to $t_{j}$ is truth; the firing of $t_{j}$, enabled by $M^{\prime}$, generates a new marking $M^{\prime \prime}$, so defined: $M^{\prime \prime}\left(p_{i}\right)=M^{\prime}\left(p_{i}\right)+O\left(t_{j}, p_{i}\right)-I\left(t_{j}, p_{i}\right)$

\section{SUPERVISOR SYNTHESIS ALGORITHMS}

In this paper the synthesis of the supervisor is obtained executing the two algorithms presented in this section. The first one is a modified version of the reachability tree algorithm presented by Murata (Murata, 1989). The second algorithm is derived from the one proposed by Ziller (Ziller and Cury, 1994).

\subsection{Modified Reachabillity Tree}

For modeling physical systems, it is natural to consider an upper bound for the number of tokens that each place can hold. A PN with this constraint is called finite capacity Petri net, and not all the markings are present in its reachabillity tree (Murata, 1989). Some information is lost when one uses the symbol $w$ to replace the markings where the number of tokens tends to grow infinitely.

Algorithm 1 Modified Reachabillity Tree Algorithm for finite capacity PNs. Begin

1. Label the initial marking $M_{0}$ as the root and tag it new;

2. While new markings exist do:

(a) Select a new marking $M$;

(b) If $M$ is identical to a marking that already exists, tag it old;

(c) If no transition is enabled at $M$, tag it as dead;

(d) While enabled transitions exist at $M$, do the following for each enabled transition:

i. Obtain the marking $M^{\prime}$ that results from firing $t$ at $M$;

ii. If there exists a marking $M^{\prime \prime}$ such that the capacity of a place is exceeded, then replace $M^{\prime}(p)$ by $w$ for each $p$ such that its capacity is exceeded;

iii. Introduce $M^{\prime}$ as a node, draw an arc with label $t$ from $M$ to $M^{\prime}$ and $\operatorname{tag} M^{\prime}$ not-permitted if the capacity of a place is exceeded, otherwise tag it new. 


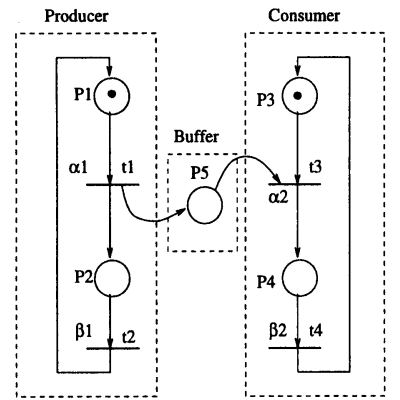

(a)

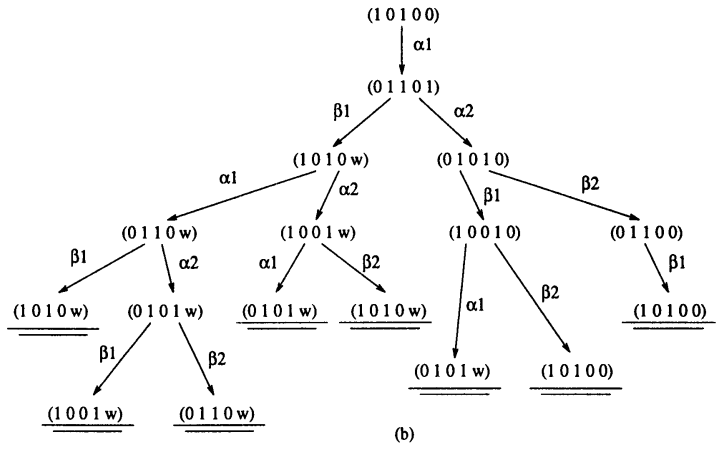

(b)

Figure 1 (a) PN model; (b) Reachabillity tree of a producer/consumer system

\subsection{Algorithm to Construct a Generator for $\sup C(L)$}

Different algorithms have been proposed to compute $\sup C(L)$. Generally these algorithms require the computation of the trim components of $G$. If the system and the specification are modeled by finite automata, then the $\sup C(L)$ exists (Ramadge and Wonham, 1989). A new algorithm to find the trim component of a system being modeled by a finite capacity $\mathrm{PN}$ and then compute the $\sup C(L)$, is introduced. To compute $\sup C(L)$ (item 5 of the algorithm) it is applied the algorithm presented by Ziller (Ziller and Cury, 1994).

Algorithm 2 Algorithm to construct a generator for $\sup C(L)$ from a $P N$ generator: Begin

1. Create a dynamic list of states, block_list, and initialize it with the blocking states (blocking markings);

2. Add to the list the states that the only output event (transition) is input of a blocking state;

3. Add to the list the states that have, at least one output transition, labeled by an uncontrollable event, that is an input transition of a blocking state;

4. Create a dynamic list of states and events, danger_list, and initialize it with the block_list ancestors (states) together with the events that link block_list states to danger_list states, since the ancestor does not belong to the block_list;

5. Given the desired system specification, compute the supremal controllable sublanguage $\sup C(L)$;

6. Add to the danger_list the states and their respective output events that will be disabled to execute the $\sup C(L)$ since these states do not belong to the danger_list.

End

\section{PRODUCER/CONSUMER EXAMPLE}

A typical producer/consumer system may be modeled by the PN of Figure 1(a). To execute a given task, it is necessary to incorporate some control action to this model. To construct the reachabillity tree of the PN generator $G$, and to derive the generator of the maximal possible specification $K^{\uparrow}$, the Algorithms 1 and 2, are executed sequentially. To 


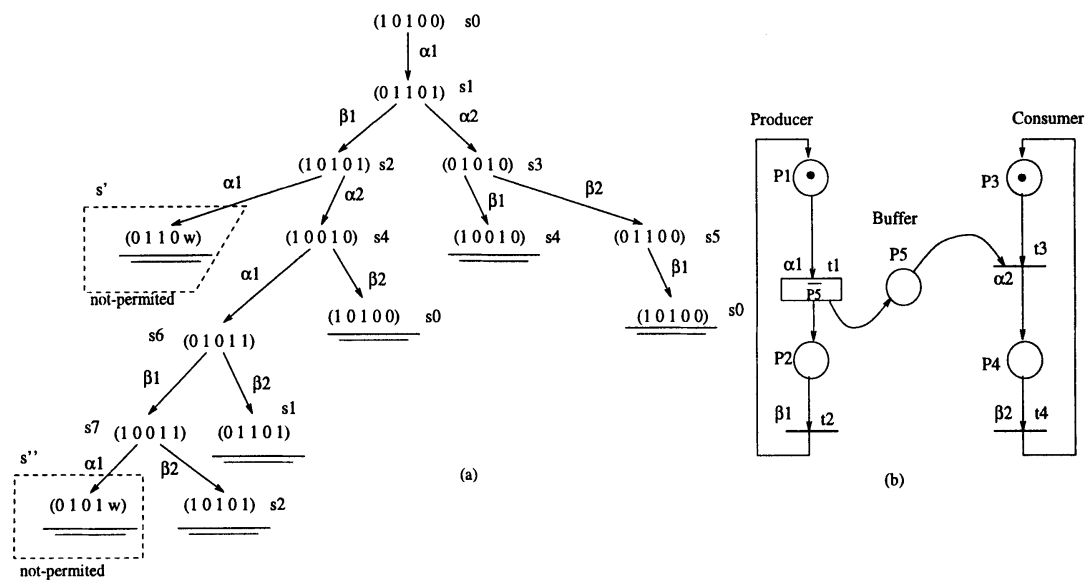

Figure 2 (a) Modified reachabillity tree of the producer/consumer PN model; (b) PNTEF supervisor for the producer/consumer system

implement the supervisor a PNTEF with the same structure of the PN model of $G$, is employed.

Considering a finite capacity buffer modeled by the place $p_{5}$, it is necessary to limit the number of tokens in that place. Observing the reachabillity tree, Figure 1(b), one can see that the number of parts produced and deposited in the buffer may become too big before one of them be consumed.

Suppose that $p_{5}$ in Figure 1(a), has capacity equal to one. Executing the Algorithm 1, the new reachabillity tree sketched in Figure 2(a), is obtained.

Notice that executing the reachabillity tree algorithm, the system state information is lost if the sequence $\left(\alpha_{1} \beta_{1}\right)^{*}$ is fired from the root (marking $\left.M_{0}\right)$. Moreover, executing the Algorithm 1, all the states where the net capacity is not exceeded, are enumerated. Besides that, one obtains information about the strings on the path from the root to a not-permitted state. Figure 2(a) shows all the physically possible strings for a system whose buffer has capacity equal to one, that is, states marked with a dashed line boxes should not be reachable. Consider the following specification: the initial state must be always reached.

To control the system, let $\sum=\left\{\alpha_{1}, \alpha_{2}, \beta_{1}, \beta_{2}\right\}$ where $\sum_{c}=\left\{\alpha_{1}, \alpha_{2}\right\}$ and $\sum_{u}=\left\{\beta_{1}, \beta_{2}\right\}$. In this case, it is necessary to disable $\alpha_{1}$ in markings $M_{2}=[10101]^{T}$ and $M_{7}=[10010]^{T}$ to achieve the desired specification.

Given the PN model of the plant $G$, and applying Algorithm 2, either the automaton that generates the language representing the desired specification may be obtained, or then its maximum possible approximation.

Applying Algorithm 2, first, the trim component of the plant $G$ is computed, and then, the generator of the least restrictive language for the desired specification. Notice that the danger_list has all the states (markings) and their respective events to be disabled. The maximum controllable sublanguage is generated by the automaton derived from the reachability tree of the Figure 2(a). The reachabillity tree of this automaton is presented in Figure 2(a). The markings in the dashed line boxes have to be excluded.

The supervisor may be implemented by the PNTEF of the Figure 2(b), i.e.: $N=$ $(P, T, I, O)$ is a PN structure, identical to the PN structure of $G ; l$ (See Section 3$)$ repre- 


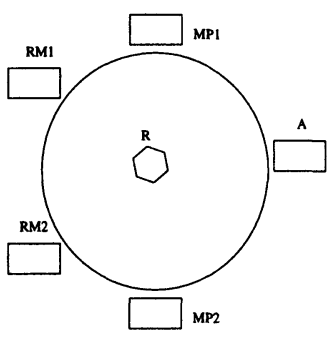

(a)

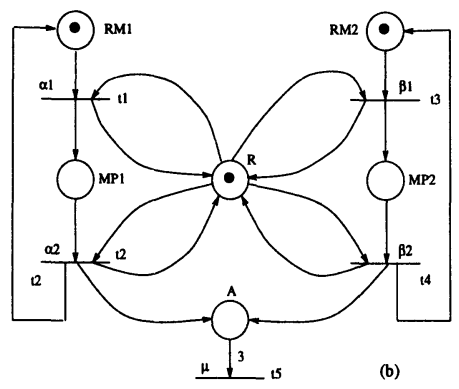

Figure 3 (a) Example of a manufacturing cell; (b) PN model of a manufacturing cell

sents the following mapping: $t_{1} \rightarrow \alpha_{1} ; t_{2} \rightarrow \beta_{1} ; t_{3} \rightarrow \alpha_{2} ; t_{4} \rightarrow \beta_{2} ; M_{0}=[10100]^{T}$ is the initial marking; $\varphi_{1}=\bar{p}_{5}$ while $\varphi_{2}=\varphi_{3}=\varphi_{4}=1$, because there are no restriction to the firing of transitions $t_{2}, t_{3}$ and $t_{4}$, since when they are enabled in some marking.

Considering that $t_{1}\left(\alpha_{1}\right)$ may not fire in two markings, only the logical expression $\bar{p}_{5}$ associated with $t_{1}$ is shown in Figure $2(\mathrm{~b})$. This means that $t_{1}$ will not be always enabled when the system is in a state that $p_{5}$ hold a token. Since the other transitions have no additional firing restrictions, the logical expressions associated to them are $\varphi_{2}=\varphi_{3}=$ $\varphi_{4}=1$, and are implicitly considered in Figure 2(b).

The logical expression $\bar{p}_{5}$ may be substituted by an inhibitor arc, and the net transformed in another net by the complementary-place transformation (Murata, 1989).

The PNTEF supervisor may be then executed in parallel with the plant, obeying to the transition firing rule.

Notice that specifying another task for the system, e.g., $K=\left(\alpha_{1} \beta_{1} \alpha_{2} \beta_{2}\right)^{*}$, it is enough to run steps 5 and 6 of the Algorithm 2. Therefore, the new states and their respective output events to be disabled are determined.

\section{A MANUFACTURING CELL}

Considering a manufacturing cell, as shown in Figure 3(a), it is possible to identify two raw-material deposits RM1 and RM2; two processing centers MP1 and MP2; and an assembling center A. A robot R moves raw-material from deposits RM1 and RM2 to the processing centers MP1 e MP2, respectively, and from these centers to the assembling center A. Figure 3(b) shows the PN model for this cell, where each three assembled parts constitute an item of this cell. Items are then transported to another cell. Exit of an item, from $\mathrm{A}$, is modeled by transition $t_{5}$ (event $\mu$ ) and is accomplished by an external agent. The maximum number of parts that the assembling center A supports is three. These parts are enough to assemble an item, i.e., these are the upper bound in the number of tokens that place A may hold in any marking of the net.

Let $\sum=\left\{\alpha_{1}, \alpha_{2}, \beta_{1}, \beta_{2}, \mu\right\}$, where $\sum_{c}=\left\{\alpha_{2}, \beta_{2}\right\}$ and $\sum_{u}=\left\{\alpha_{1}, \beta_{1}, \mu\right\}$. After executing Algorithm 1, the modified reachabillity tree for the PN model of the manufacturing cell, is obtained. This tree is shown in Figure 4.

The automaton, shown in Figure 5, represents a specification to obtain a given item. An item is assembled with two parts processed by MP1 and one processed by MP2. It is necessary to assemble first parts processed by MP1.

Executing the Algorithm 2, the generator of the $\sup C(L)$ is obtained (In this case, the same automaton of the specification). See Figure 5. The Algorithm provides the 


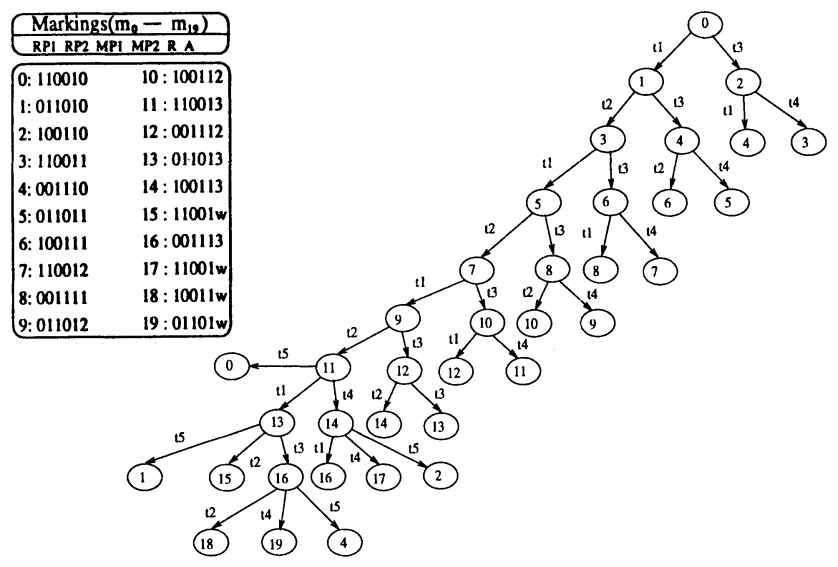

Figure 4 Reachabillity tree for the system with capacity three

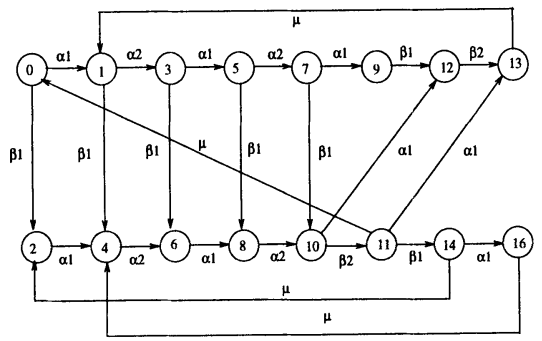

Figure 5 Automaton generator of the specification

danger_list, i.e., the list of states and respective output events to be disabled in that states or markings. See Figure 6(a). The supervisor is then shown in Figure 6(b). Notice that, given the danger_list, Figure $6(\mathrm{a}), t_{2}\left(\alpha_{2}\right)$ may fire only when the number of tokens in the place $\mathrm{A}$ is less or equal to $1(\mathrm{~A} \leq 1)$. In the same way, $t_{4}\left(\beta_{2}\right)$ may fire only when the place $A$ holds a number of tokens equal to $2(A=2)$. In any other situation, these transitions will be disabled, as shown by the respective associated logical expressions.

\section{CONCLUSIONS}

This paper introduced an extended Petri net model with transition enabling functions. This extension was used in modeling and synthesis of supervisors. A modified reachabillity tree algorithm was also introduced. This algorithm allows obtaining all the markings in a not modified finite capacity PN model. Finally, an algorithm to the construction of a $\sup C(L)$ generator was introduced. This algorithm is executed based on the modified reachabillity tree of the system PN model, and enumerates all the states and respective output events to be disabled.

Using the PNTEF model and the enumerated algorithms, it was proposed a systematic approach to solve the supervisory synthesis problem, with no modification in the structure of the net that models the system. On the other hand, this approach do not solve the 


\begin{tabular}{|c|c|c|c|c|c|}
\hline $\begin{array}{l}\mathrm{t} 2 \\
(\alpha 2)\end{array}$ & 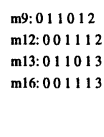 & $M(A) \leq 1$ & $\begin{array}{l}\mathrm{t} 4 \\
(\beta 2)\end{array}$ & $\begin{array}{l}\mathrm{m} 2: 100110 \\
\mathrm{~m} 4: 001110 \\
\text { m6: } 100111 \\
\text { m8: } 001111 \\
\text { m14: } 100113 \\
\text { m16:001113 }\end{array}$ & $M(A)=2$ \\
\hline
\end{tabular}

(a)

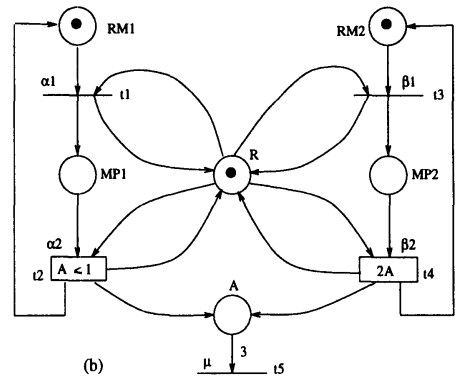

(b)

Figure 6 (a)Table of markings and their respective events to be disabled; (b) PNTEF supervisor of the manufacturing cell

classic state explosion problem of DESs, once the modified reachabillity tree has been employed. To solve this problem one possible solution is to employ a modular approach for the analysis and synthesis of the controller. Actually the use of G-Nets (Perkusich, 1994) as a modular formalism is under investigation.

\section{REFERENCES}

Giua, A. and F. DiCesare (1994). Blocking and controllability of petri nets in supervisory control. IEEE Transactions on Automatic Control 39(4), 818-823.

Murata, T. (1989). Petri net: properties, analysis and applications. In: Proceedings of the IEEE - vol. 77, num. 4. pp. 541-580.

Papelis, Y.E. and T.L. Casavant (1992). Specification and analysis of parallel/distributed software and systems by petri nets with transition enabling functions. IEEE Transactions on Software Engineering 18(3), 252-261.

Perkusich, A. (1994). Analise de Sistemas Complexos Baseada na Decomposiç ao de Sistemas de G-Nets. PhD thesis. Universidade Federal da Paraíba - Campus II. Campina Grande, Paraíba.

Ramadge, P. J. G. and W. M. Wonham (1989). The control of discrete event systems. Proceedings of the IEEE 77(1), 81-97.

Sreenivas, R. S. (1993). A note on deciding the controllability of a language $\mathrm{k}$ with respect to a language l. IEEE Transactions on Automatic Control 38(4), 658-662.

Ziller, R. M. and J. E. R. Cury (1994). On the supremal l-controllable sublanguage of a non prefix-closed language. In: Anais do 10. Congresso Brasileiro de Automática, Rio de Janeiro. pp. 260-265.

Giovanni C. Barroso received the B.S. degree in EE from UFC, Fortaleza, CE, Brazil, in 1982, the M.Sc. degree in EE in PUC/RJ, Rio de Janeiro, RJ, Brazil in 1987. He is a faculty member of DF/UFC, since 1989. He is a Ph.D. candidade at UFPB, Campina Grande, PB, Brazil.

Antonio M.N. Lima received the B.S. and M.S. degree in EE from UFPB, Campina Grande, PB, Brazil in 1982 and 1985 respectively, and doctaral degree in EE from INPT, Toulose, France, in 1989. He is a faculty member of DEE/UFPB since 1983.

Angelo Perkusich received the B.S. degree in EE, from UNESP, Barretos, SP, Brazil, in 1982, the M.S. and doctoral degree in EE from, UFPB, Campina Grande, PB, Brazil in 1986 and 1994 respectively. He is a faculty member of DEE/UFPB since 1991 . 